\title{
ESTUDIO DE LAS ALGAS RHODOPHYTA DEL LITORAL DE TACNA (BOCA DEL RIO, VILA-VILA Y CANEPA)
}

Juan Franco León', César Acleto Osorio²,

Vicente Chambilla Quispe ${ }^{3}$, LiduvinaSulcaQuispé.

\section{R E S U M E N}

En el estudio realizado sobre la distribución sistemática de las Rhodophytas del litoral rocoso en las localidades de Boca del Rio, Vila-Vila y Cánepa, (playas de Tacna), de abril 92 a junio 94 , se identificaron 16 especies de algas rojas y muestras todavía no reconocidas. Se empleó el criterio de zonación de paredes, en el cual la zona intermareal se extiende desde los niveles de bajamar a pleamar, observándose cinturones horizontales, representados por agrupaciones de fauna y flora.

Las especies identificadas son:Porphyra columbina; Porphyra pseudolanceolata; Grateloupia dorypora; Corallina officinalis var chilensis; Gymnogongrus furcellatus; Gigartina glomerata; Montemaria horridula; Rhodymeria skottsbergii; Gastrodonium pacifium; Streblodadia camptodada; Centroceras claucelatum; Ceranium rubrum; Chondrus canaliculatus; Trematocarpus didrotomus; Chaetangium fastigiatum; Pterosiphonia dendroidea.

\section{INTRODUCCIÓN}

Las algas marinas, generalmente, son organismos Iturales, sometidos a la acción de las corrientes acuáticas de modo considerable. Por lo común, viven adheridas a sustratos rocosos, notándose claramente la diversidad de especies en relación al sustrato y profundidad marina en que se desarrollan.

A lo largo de nuestra costa se puede apreciar una buena diversidad de algas marinas, que sugieren la idea de un aprovechamiento industrial sobre la base de un conocimiento básico preciso y acorde al avance de la tecnología.

En la actualidad, en algunas partes del mundo, se le da mucha importancia y aprovechamiento a este recurso marino como alimento, forraje, fertilizante, uso medicinal y principalmente en la industria, por las diversas utilidades que se da a los ficocoloides que se extraen de estos organismos, como son: el agar-agar, alginatos, canagenanos, etc. Así, algunos de estos organismos pueden comportarse como indicadores de contaminación de las algas marinas superficiales.

1. Biólogo

2. Doctor en biología

3. Bachiller en ciencias biológicas
La presente investigación pretende dar a conocer y difundir las características ecológicas, distribución, hábitat y sistemática de las Rhodophyta de nuestro litoral, considerando las cuatro estaciones del año.

\section{MATERIAL Y MÉTODO}

El trabajo de investigación se realizó en el litoral rocoso del departamento de Tacna, considerando tres lugares de muestreo: Boca del Río, caleta de Vila-vila y playa Cánepa, ubicándose en las coordenadas geográficas siguientes $70^{\circ} 48^{\prime} 36^{\prime \prime}$ longitud oeste, $18^{\circ} 06^{\prime} 30^{\prime \prime}$ latitud sur, $70^{\circ} 32^{\prime} 35^{\prime \prime}$ Iongitud oeste y $18^{\circ} 06^{\prime} 00^{\prime \prime}$ latitud sur.

De las tres zonas de trabajo, la caleta de Vila-Vila constituye un centro principal de desembarque de recursos hidrobiológicos del litoral tacneño, perteneciente al distrito de Sama. Asimismo, estas 3 zonas se ubican a $60,9 \mathrm{~km}$ de distancia de Boca del Río, $67 \mathrm{~km}$ de la caleta de Vila-Vila y $69 \mathrm{~km}$ de playa Cánepa, y están comunicadas con Tacna a través de una amplia carretera asfaltada.

El litoral de Tacna geográficamente presenta características bien definidas que se pueden clasificar 
en dos tipos de costa: arenosa y rocosa. Las costas arenosas comprenden desde el límite fronterizo La Concordia hasta el balneario de Llostay. Se presentan en forma llana y longitudinalmente recta, mientras que las costas rocosas comprenden desde Llostay hasta el límite con el departamento de Moquegua, manifestándose, además, algunas zonas arenosas.

El material estudiado corresponde a la colección realizada, durante los años 1992-1994. Se encuentran en gran parte, conservados en una solución de formol al $5 \%$ con agua de mar y también herborizados, integran en la actualidad la ficoteca del laboratorio de Botánica de la U.N.J.B.G. de Tacna.

La identificación se ha realizado medianteel estudio morfológico por comparación y cortes histológicos de las muestras utilizando láminas, claves taxonómicas y descripciones de la especialidad.

\section{DESCRIPCION: RHODOPHYTA (Boca del Río - Vila Vila)}

\section{Clase : Bangiophycidae \\ Orden : Bangiales \\ Familia: Bangiaceae \\ Género : Porphyra C. Agardh \\ Especie: Prophyra columbina Montagne (fig. 1)}

Algas generalmente saxícolas, de color pardo amarillento, púrpuraclaro o rojizo de $8-40 \mathrm{~cm}$ de longitud; disco coriáceo parenquimático, constituido por células filamentosas de $11,5 \mathrm{~mm}$ dediámetro. Tallosmonoatro máticos, laminares membranosos, moderadamente elásticos longados, lineares, lanceolados, bordes crenados o irregularmente denotados, ondulados 0 crispados, de 1-8 cm de ancho.

El patrón de distribución geográfica de esta especie está asociado comúnmente con otros organismos (conchas de choros, otras algas, etc.). Se distribuye desde el norte peruano: Paita, Trujillo, Lima, Arequipa, Tacna, Coquimbo-Chile. Vive en los niveles altos de roqueríos inter-mareales.

\section{Especie: Porphyra pseudolanceolata Montagne (fig. 2).}

Especie de color púrpura claro, ligeramente rojizo, tallos lanceolados, presenta disco coriáceo parenquimático, bordes muy irregularmente denotados, a ondulados, de unos $1,5-2 \mathrm{~cm}$ de ancho. Comúnmente también se le puede hallar asociado con otros organismos. Su distribución se registra desde el norte peruano hasta el sur de Chile, vive en los niveles altos de roqueríos inter-mareales.

\section{Clase : Floridisphycidae \\ Orden :Cryptonemiales}

\author{
Familia: Cryptonemiaceae \\ Género : Grateloupia c. Agardh \\ Especie: Grateloupia doryphora Howe \\ (fig. 3)
}

Alga que comúnmente es abundante en ámbitos protegidos y casi siempre expuesta al oleaje, preferentemente tiende a desarrollarse sobre rocas en mareas bajas y pozas. Presenta un carácter estacional, una marcada variación morfológica, en la cual la fronda puede ser ancha y suborbicular o angosta, alargada y lanceolada. En pocas y muchas proliferaciones. Puede llegar a medir desde $30-40 \mathrm{~cm}$ de longitud y $20 \mathrm{~cm}$ de ancho, fronda de textura suave, de un color rojo púrpura,rojo-pardo, algunas veces con tonos verdosos. Estípite cilíndrico cerca de la base. Se le considera como una Rhodophyta endémica del Pacífico, desplazándose desde Paita hasta Tierra del Fuego.



Esta especie es una alga calcárea articulada, pudiendo dominar diferentes hábitats.Por ejemplo, puede de desarrollarse en pozos y piletas litorales, frecuentemente en frentes expuestos al oleaje, donde adquieren mayor tamaño. Sus frondas son rosadas, algunas veces pálidas. Su tamaño puede llegar a $15 \mathrm{~cm}$ de longitud. Muestran un eje principal ramificado lateralmente en forma bipinada. Su biomasa es muy abundante en relación con otras especies. Su distribución es amplia en mares templados y fríos. Se desplaza desde Tierra del Fuego, Arica, Tacna hacia el norte del Perú.



Se ubica en zonas bajas de rocas que comúnmente sufren invasión de arena.

Aparece como mechones gruesos, ligeramente grandes de hasta $15 \mathrm{~m}$ de altura, muy ramificado. Color preferentemente púrpura, la porción terminal es de color rosado o de color rojo claro. Su ramificación es dicotómica en forma repetida; los segmentos basales son más largos que los apicales, pudiendo alcanzar $3 \mathrm{~cm}$ de longitud por $2 \mathrm{~mm}$ de ancho.

Su clasificación es desde Arica-Chile hasta TacnaPerú, hacia el norte. En sitios como Nueva Zelandia y sur de Australia, también se reporta su presencia.

Familia : Gigartinacea

Género : Gigartina Stackhouse

Especie : Gigartina glomerata Howe.

(fig. 6) 
Algas comúnmente cespitosas, algunas veces gregarias. Su color es de marrón-verdoso o marrónrojizo. También de $3-12 \mathrm{~cm}$ de longitud, disco amplio calcáreo, tallo cartilaginoso, algo comprimido. El diámetro que presenta el tallo va de $1-5 \mathrm{~mm}$, pudiendo ser erectos o incurvos. Su ramificación es dicotómica. Presenta espinas cor'?s, agudas. Muchas veces, en los tallos pueden presentarse proliferaciones muy cortas. Su estructura reproductora compuesta por tetrasporangios ovoides ligeramente clauiformes, dispuestos en sacos superficiales, redondeados, alargados, semilunares o irregulares. Cistocarpos esféricos o semi-esféricos superficiales.Su distribución se reporta desde Trujillo a Tacna.

\section{Familia : Sphaerococcaceae Género : Montemaria Especie : Montemaria horridula Yoly et Alveal (fig. 7)}

Estas algas forman penachos y cojines (algunas veces a manera de césped) de color rojo oscuro, rojopúrpura. Habitan pozas de mareas y superficies rocosas, casi siempre expuestas al oleaje. Presentan hapterios, por medio del cual se adhieren a las rocas; estos últimos tienen forma de disco originado por un eje cilíndrico postrado y rastrero. El tamaño que presentan puede llegar a los $2 \mathrm{~cm}$ de alto con su extremo distal y proximal estrechado con la porción media claramente ensanchada. Tanto las ramificaciones y la porción apical, están investidas con ramificaciones cortas aguzadas que, parecen espinas.

Están comúnmente asociadas con otras algas de una condición perenne.Su distribución reportada para el sur, en Chile se le considera como endémica (Valparaíso y Coquimbo), en Tacna, se presenta en forma abundante.

\section{Orden : Rhodymeniales \\ Familia : Rhodymeniaceae \\ Género : Rhodymenia \\ Especie : Rhodymenia skottsbergii Dawson (Fig. 8)}

Son algas gregarias, alcanzan hasta $5 \mathrm{~cm}$ de altura. Su coloración puede variar de marrón-violáceo a rosado; presentan rizoides discoidales simples a ramificados. Sus ramas son de aspecto laminar, con terminación dicótoma, flabeladas alternas, orientadas en ángulo recto; su anchura puede variar de 4-5 mm. Los cistocarpos s obresalen muy notoriamente sobre la superficie media de las láminas próximas, al ápice en ambos lados. Los tetrosporangios se ubican en la zona cortical de las láminas marginales. Es común en niveles bajos de la zona de mareas y en hábitats submareales hasta $70 \mathrm{~cm}$ de profundidad.

Comúnmente viven en grupos sobre substrato rocoso. En la zona de las mareas de nuestro registro, amplía su distribución geográfica indicada antes para las costas de Chile, Tacna y parte del norte.

\section{Familia : Champiaceae \\ Género : Gastroclonium \\ Especie: Gastrodonium pacifium \\ Santalices (fig. 9)}

Esta especie es pequeña, con tallo cilíndrico ramificado; los ramos mayores son muy cortos. Su coloración es variable, aunque es frecuente el verdoso-marrón claro. Puede llegar a medir $1-3 \mathrm{~cm}$ de largo por 1-1,5 mm de ancho. Comúnmente se desarrolla en áreas sombrías, mezclada con otras algas.

Su distribución está reportada para Arica y Valparaíso (Chile); en el Perú, Tacna.

\section{Orden : Ceramiales \\ Familia : Rhodomelaceae \\ Género : Streblocladia \\ Especie : Streblodadia camptodada Falkenbreg (fig. 10)}

Alga que puede ser encontrada como epífita; su longitud aproximada es de $2-5 \mathrm{~cm}$ y con unos tallos comprimidos o ligeramente cilíndricos de 340-350 u de diámetro. Su color varía entre el rojo-oscuro y el marrónclaro.

Presenta discos adhesivos; ramificación profusa, simpódica, conterminales generalmente laxas, tomando muchas veces el aspecto de un repollo. Tetros porangios esféricos, dispuestos en series adaxiales, o escasos en el extremo de las ramas de 42-56 u de diámetro; cistocarpos Pixiformes, coronados, pedicelados, seriados o alternos de 520-617 u de largo por 192-393 $\mathrm{u}$ de ancho.

Comúnmente se encuentra en zona intermareal o submareal. Su distribución se reporta para el Perú desde Talara-Mollendo-Tacna.

\section{Familia: Ceramiaceae \\ Género: Centroceras \\ Especie: Centroceras claucelatum(Ag.) Montagne (fig. 11)}

Especie muy común en los niveles medio y alto de la zona de mareas de Chile central y sur peruano (Tacna). Forma el aspecto de mechones y penachos de 3-5 cm de alto. Su coloración va desde el rojo oscuro al púrpura, constituido por filamentos finos, flexibles y delicados; estos filamentos miden de 150-200 m de diámetro; son segmentados con internados de 300-600 $\mathrm{m}$ de largo. Su corteza está formada por células pequeñas, dispuestas formando corridas longitudinales. Las últimas ramificaciones son incurvadas y semejan los brazos de una periza curva. Tiene una amplia distribución en Chile, hasta el Perú.

\section{Género : Ceramium \\ Especie: Ceramium rubrum (Huds) Agardh (fig. 12)}

Planta marina muy corta, de $5 \mathrm{~cm}$ de alto. Se desarrolla en la zona media de mareas, constituyendo 
pequeñas matas filamentosas, muy cargadas, de color rojo oscuro o rojo púrpura. Su característica típica es que sus filamentos son delgados y flexibles, siendo un poco más firmes que los de otras especies de este mismo aspecto, tamaño y morfología. Los ejes son ramificados en forma dicotómica, y se van haciendo más estrechos hacia los ápices. Los segmentos son cortos en las porciones apicales de las plantas y dos veces más largos que anchos, en las porciones medias de la planta.

La especie presenta una amplia distribución en aguas templadas. Se reporta desde Tierra de Fuego, Antofagasta-Chile al sur peruano (Tacna).

\section{Orden : Gigartinales \\ Familia : Gigartinacea \\ Género : Chondrus \\ Especie : Chondrus canaliculatus \\ (Ag.) Greville (fig. 13).}

Algas que se desarrollan sobre rocas, en zonas intermareales medias y bajas, en frentes expuestos o semiexpuestos al oleaje. Su coloración es de rojopardo, rojo-púrpura, rojo carmín.

Las plantas miden hasta $14 \mathrm{~cm}$ de alto,dividiéndose en forma dicotónica. Los segmentos son fastigiados, los más anchos son claramente coniculados 0 débilmente cóncavo-convexos, el área donde se originan las dicotómicas puede llegar a medir $2 \mathrm{~cm}$ de ancho y $1 \mathrm{~mm}$ de grosor. Seadelgazan haciala porción más basal, donde rematan en un estipite cilíndrico; también es común encontrar plantas cistocárpicas, en cuyo caso los cistocarpos aparecen como pequeñas verrugas en el lado convexo de la fronda.

La especie es endémica de la costa temperada del Pacífico de Sudamérica y se reporta desde Pisco, Tacna en el Perú, continuando hasta Chile.

\section{Familia : Sarcodiaceae \\ Género : Trematocarpus \\ Especie : Trematocarpus didrotomus Kutzing (fig. 14)}

Esta alga vive,preferentemente, en grietas y pozones bajos de la zona de mareas sobre rocas, a las que seadhiere pormedio de un disco adhesivo redondo y pequeño que desarrolla su Rizoide.

Tallo cartilaginoso, comprimido o aplanado, flabelado con fo ma de abanico de hasta $10 \mathrm{~cm}$ de alto, toma una coloración rojo-pardo a pardo-oliváceo. Tallo dividido dicotómicamente en forma repetida. En sus partes más anchas puede medir hasta $5 \mathrm{~mm}$ de ancho por $200 \mathrm{~m}$ de espesor.

Realizando un corte transversal, se puede apreciar una médula constituida por filamentos dispuestos en forma laxa y una corteza formada por varias capas de células; se le considera como una alga endémica de sudamérica, desde la bahía de Paracas-Perú hasta el sur de Chile.

\section{Orden : Nemaliales \\ Familia : Chaetangiaceae \\ Género : Chaetangium \\ Especie : Chaetangium fastigiatum \\ (Bory) Agardh (fig. 15).}

Alga erguida, cartilaginosa, comprimida; de color marrón oscuro a marrón negro, alcanza hasta $6 \mathrm{~cm}$ de altura, con ramificaciones dicotómicas 4-8veces, ramas con la porción basal angosta de $1 \mathrm{~mm}$ de diámetro, luego se hace más amplia y alcanza hasta $0,8 \mathrm{~cm}$; el ápice es acuminado y termina en punta aguda o redondeada. Son plantas dioicas: las femeninas son más robustas y menos ramificadas, se caracterizan por presentar los cistocarpos globosos abundantes en la parte media de las ramas, embebidas entre la capa cortical y los filamentos medulares.

Las plantas masculinas se diferencian muy ligeramente delas femeninas, por ser éstas mucho más ramificadas y de ramas angostas. Los espermacios se presentan distribuidos sobre el tallo, generalmente en grupos, formando un cenaptáculo amplio y poco profundo.

Preferentemente están en zona intermareal y submareal. Su distribución se reporta en Chile, sur y norte peruanos.

\section{Orden : Ceramiales \\ Famili : Rhodomelaceae \\ Género : Pterosiphonia \\ Especie: Pterosiphonia dendroidea (Roth)Falkenberg(fig.16).}

Algas epífitas, con ertolón de color rojo-oscuro a rojo vinoso, de $2-8 \mathrm{~cm}$ de longitud. Presentan discos adhesivos circulares; tallos generalmente comprimidos, cilíndricos o triangulares de 400 a 800 u. de diámetro, Ramificación con pinnas percurrentes más largos que los superiores; ápices incurvos oligeramente circinados, raras veces rectas.

Generalmente son intermareales a submareales, también viven asociados con otras algas. Son de aspecto frágil, lo que requiere cuidado en su colecta.

Su distribución reporta desde Paita, Trujillo, Tacna, hacia el sur chileno

\section{Discusión}

El inventario sistemático de las comunidades de macroalgas, en la zona intermareal rocosa de las tres áreas de muestreo, está representado por 16 especies de Rhodophytas, en las que se incluyen 2 para la subclase Bangiophycidae y 14 para la subclase Floridiophycidae.

En relación con otros autores, se puede mencionar a Bustamante (1983), investigador que reporta 16 especies, 10 órdenes, 4 clases y 4 divisiones, incluyendo 
a la División Cyanophyta; de todas ellas sólo 2 especies son comunes con el presente trabajo: Porphyra columbina Montagne; Corallina officinales var. chilensis. Kutzing.

La investigación realizada por Calla et al (1990), sobre algas marinas de la bahía de Catarindo, reporta 20 especies distribuidas en 3 Divisiones; cuatro Chlorophyta; cinco Phaeophytas; y once Rhodophyta; coincidiendo al igual que la presente investigación en presentar a $C$. officinalis var. chilensis como la más abundante; también determinó a Grateloupia donyphora Howe, Rhodymenia skottsbergil Falk. y Pterosiphonia dendroidea Falk

En 1983 Barnigo, trabajando en Catarindo, reportó 15 especies, de las cuales 6 especies de rhodophytas son comunes entre nuestra zona y la de Catarindo. Porphyra columbina Montagne; Corallina officinalis var. chilensis, Kutzing; Gigartina glomerata Howe; Montemaria horridula Jocy et Alveol; Rhodymenia skottsbergii Dawson; Centroceras clovulatum Mont.

Vargas, trabajando en 1994 (en Catarindo, Mejía y Quilca) menciona una similitud en lo referente a Rhodophyta, caracterizándose la elevada presencia de $C$. officinalis var. chilensis.

Por último, Acleto, desde muchos años atrás, principalmente en su publicación sobre Aspectos Fitogeográficos y Taxonómicos de las Algas Marinas del Perú, proporcionó un registro de 228 especies para la costa del Pacífico del Perú con un total de 160 Rhodophytas

\section{REFERENCIAS BIELIOGRAFICAS}

Acleto, 1973. Algas Marinas del Peru Boletin de la Socidad Peruana de Botánica, vol. VI No. 1 y 2. 1986, Algas Marinas del Perú de importancia económica . UNMSM. Museo de Historia Nacional "Javier Prado".

1988 Aspectos Fitogeográficos y Taxonómicos de las Algas Marinas del Perú. Museo de

Histotia Natural Javier Prado. GayanaBot. 45. Acosta J. 1977 Las Algas Marinas de la Provincia de

Pisco. Museo de Historia Natural "Javier Prado Barriga R. 1993. Fvaluación y Estructura

Vegetacional de Macroalgas Marinas del

Litoral de la Provincia de Islay - Arequipa.

FacultaddeCienciasBiológicasyAgropecuarias, Universidad Nacional San Agustin ,Tesis.
Calla, A., Villegas, J.y otros.1990.Algas Marinas de la Bahia de Catarindo. II Congreso de Ficologia Marina ; Universidad Federico Villarreal .

Clinton, D. 1986 Botánica Marina. Edit. Limusa, Mexico.

Fernández, A. 1987. Botánica Criptogánica. Universi dad Nacional de Trujillo.

Paredes, C. 1974. El modelo de zonación en la orilla rocosa del departamento de Lima. Revista Peruana de Biología, UNMSM.

Santelices, B. 1988. Investigaciones de las Algas Ma rinas de Chile. Fac. de Cs. Biológicas, Universi dad Católica de Chile.

Vargas, M. 1994. Distribución vertical de Macroalgas Marinas en el Intermarial del Litoral Rocoso deCatarindo, Mejia y Quilca (agosto 1993enero 1994). Tesis.

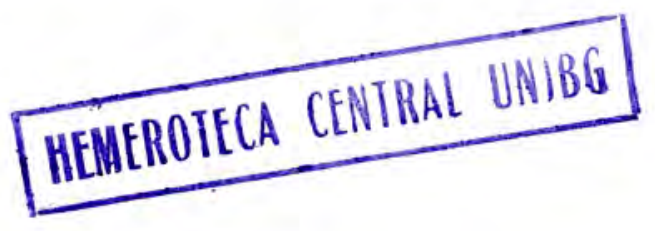




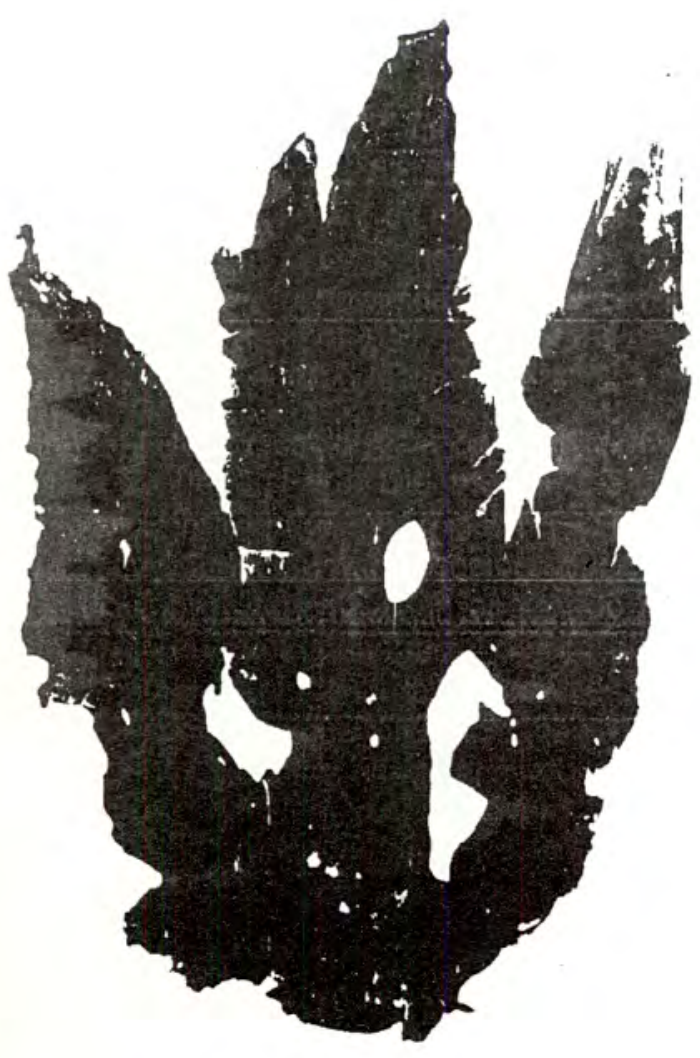

Fig. 1 Porphyra columbina Montagne

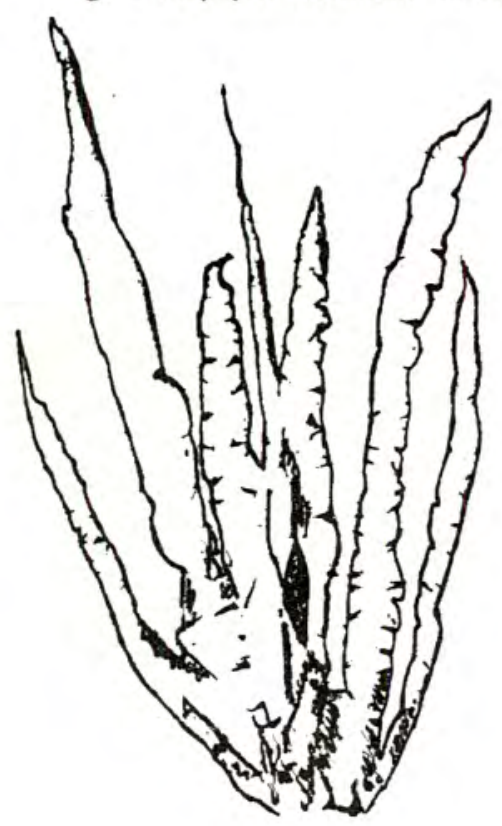

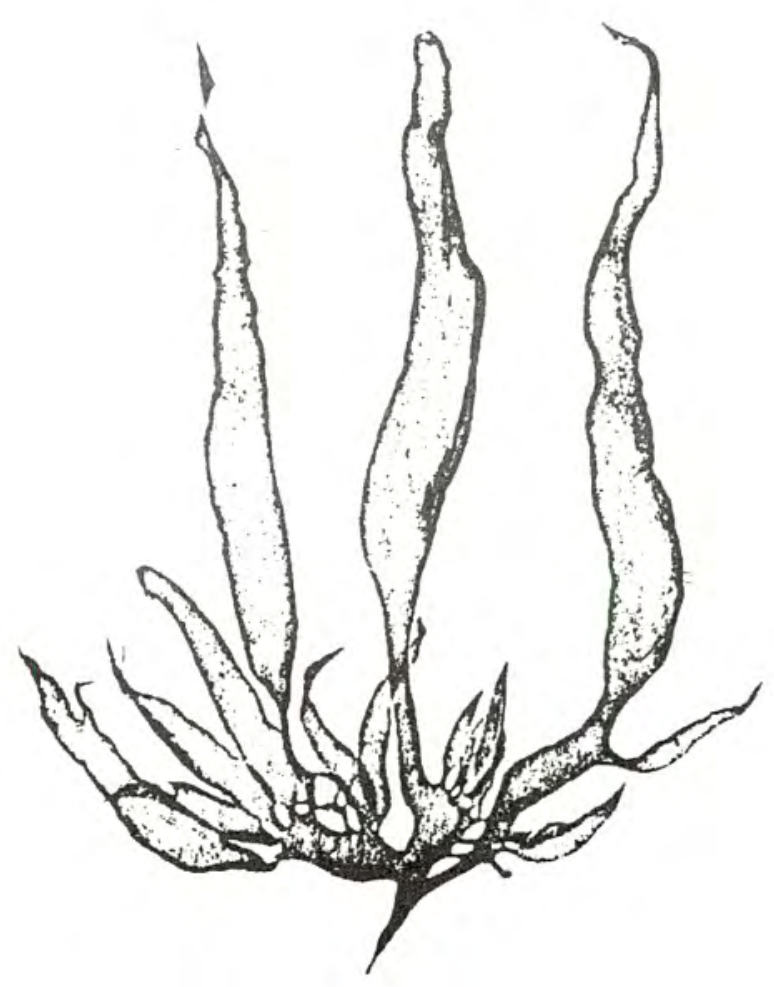

Fig. 3 Grateloupia doryphora Howe

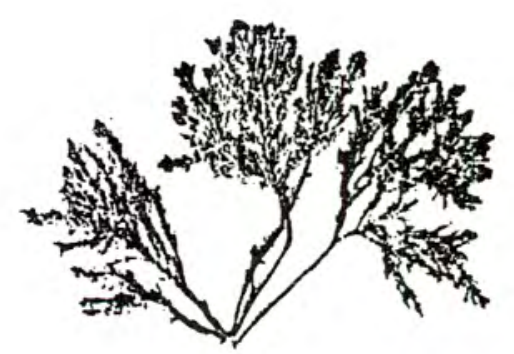

fig. 4 Corallina officinalis var. chilensis Kuts

Fig. 2 Porphyra pseudolanceolata Montagne 




Fig. 5 Gymnogongrus furcellatus Agardh

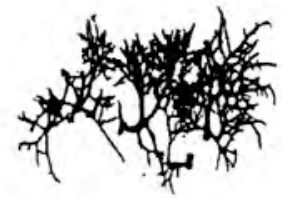

Fig. 6 Gigartina glomerata Howe

Fig. 9 Gastroclonium pacificum Santalices
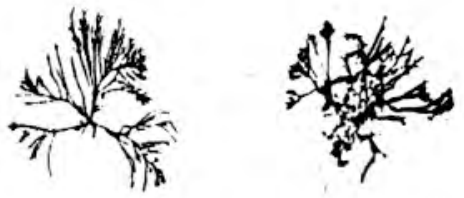

Fig. 7 montemaria horridula Joly et Alveal
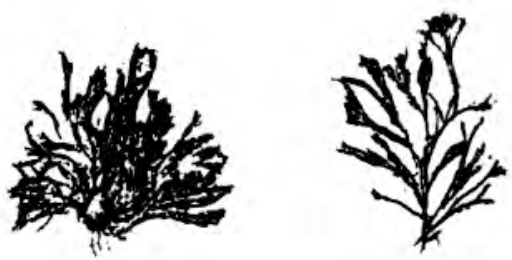

Fig. 12 Ceramiun rubrum ( Huds) Agardh

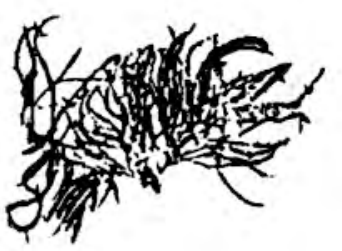

Fig 8 Rhodymenia skottsbergii Dawson

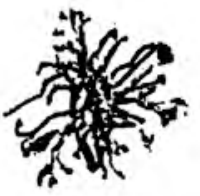

Fig. 10 Streblocladia camptoclada (mont) Falkenbreg



Fig. 11 Centroceras clavulatum Montagne

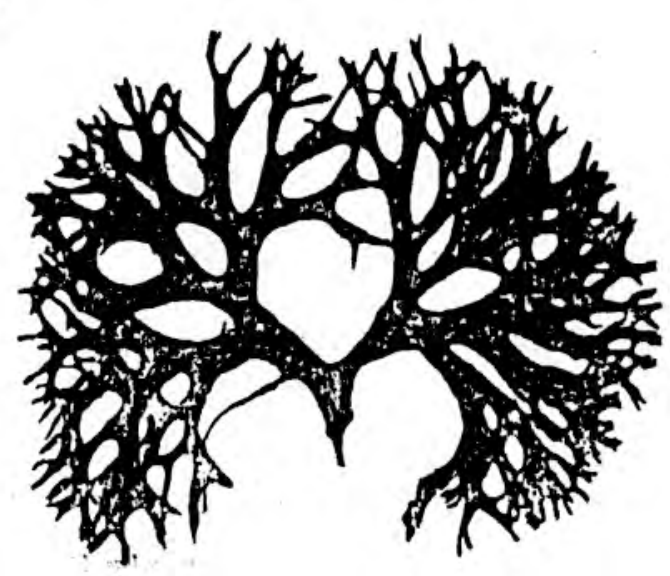

Fig. 13 Chondrus canaliculatus Greville 